\title{
Spotted around the web: Neuronal differentiation, vitamin D, Hollywood representation
}

\author{
BY JILL ADAMS, ANGIE VOYLES ASKHAM, LAURA DATTARO, PETER HESS, NIKO \\ MCCARTY
}

30 JULY 2021

WEEK OF JULY $26^{\mathrm{TH}}$

\section{Research roundup}

- Neurons differentiate prematurely in human brain tissue with FOXG1 mutations, which lead to a syndrome characterized by intellectual disability and epilepsy, supporting findings in animals. European Journal of Medical Genetics

- Mice carrying mutations in the TSC1 gene, which has been associated with autism traits, show altered development of inhibitory neurons and impaired social behavior. Nature Communications

- Autistic adults who say their work ability is poor also tend to have social anxiety and problems switching among tasks. Autism

- Postmortem studies of autism typically find alterations in the brain's frontal cortex and cerebellum. Neuroscience \& Biobehavioral Reviews

- Gene variants that alter histone function - and thereby the regulation of cellular DNA - are linked to autism; confirming the association, mice missing the SUV39H2 gene are hyperactive and show inflexibility on a complex learning task. Molecular Psychiatry

- Children with more autism traits tend to have smaller brain volumes and, in some regions, thinner cortical layers and less gyration than children with fewer autism traits. Autism Research

- Neonatal oxytocin administration appears to alter hippocampal development and improve social behavior. Molecular Psychiatry

- Advances in precision medicine put new and better treatments for autism in reach, according to a new commentary. Neuron

- Two new publicly available algorithms can accurately predict protein structures from DNA 


\section{Spectrum | Autism Research News}

https://www.spectrumnews.org

sequences. Nature

- Genetic characterizations of autism have far outpaced phenotypic ones; however, synthesizing the two may be necessary to understand autism, argues Isaac Kohane. Neuron

- Repetitive behaviors observed in first-degree relatives of people with autism or with obsessive compulsive disorder show similar patterns, strengthening the idea that a common pathway underlies both conditions. Autism Research

- Low levels of vitamin D during a woman's pregnancy are associated with higher odds of autism in her child; Spectrum has reported on similar associations found in larger studies. Biological Psychiatry

- Autistic boys and girls have bigger sensory processing differences than non-autistic boys and girls do. Autism Research

- A prolonged-release form of melatonin improves sleep onset and duration in autistic children with insomnia. Expert Opinion on Pharmacotherapy

\section{Science and society}

- The U.K. government has announced an Autism Strategy, which includes funding to improve diagnosis and access to treatment and educational settings for people on the spectrum. UK

- Autism and disability advocates say they are underrepresented in Hollywood, which casts non-disabled actors as disabled characters and tells stories that rely on disability stereotypes. Ms. Magazine 